\title{
Regulated Histone Methyltransferase and Demethylase Complexes in the Control of Genes by Nuclear Receptors
}

\author{
A. Yokoyama, R. Fujiki, F. Ohtake, and S. Kato \\ Institute of Molecular and Cellular Biosciences, University of Tokyo, Bunkyo-ku, \\ Tokyo 113-0032, Japan \\ Correspondence: uskato@mail.ecc.u-tokyo.ac.jp
}

\begin{abstract}
Liganded nuclear receptors (NRs) are DNA-binding transcription factors that control the transcription of target genes. Such NRs exert their transcriptional functions via ligand binding-induced interactions with a number of coregulator complexes to reorganize chromatin state. Intensive investigation of NR coregulator complexes has revealed that, besides histone acetylation, histone methylation is critical for ligand-dependent transcriptional controls by NRs. Our recent biochemical screening for NR coregulator complexes showed that the enzymatic activities of these histone methylation/demethylation complexes are under the control of posttranslational modifications (PTMs) of their catalytic subunit. Characterization of such regulated complexes has established the concept that transcriptional coregulator complexes sense and decode cellular signals at the molecular level. In this symposium review, we will illustrate our recent findings regarding PTM-based regulation of NR transcriptional control and discuss how these findings are applicable to the diverse roles of NR coregulators in interpreting regulatory signals into proper gene regulation.
\end{abstract}

Nuclear receptors (NRs) form a gene superfamily, and the group of liganded NR serve as ligand-dependent transcription factors in response to steroid/thyroid hormone, vitamin $\mathrm{A} / \mathrm{D}$, and other fat-soluble ligands (Mangelsdorf et al. 1995). The other NR members are orphan receptors, and act as constitutively active/repressive transcription factors (Giguere 1999). Through such transcriptional control of target genes, NRs play a wide variety of roles in diverse biological events (Bamberger et al. 1996; Couse and Korach 1999). NR superfamily members are defined by the presence of a highly conserved DNAbinding domain (DBD) and a less conserved carboxyterminal ligand-binding domain (LBD) (Mangelsdorf et al. 1995). To date, $48 \mathrm{NR}$ genes have been identified in the human genome, and the NR superfamily is conserved throughout the metazoans (King-Jones and Thummel 2005).

Like the other classes of DNA-binding transcription factors, NRs require the fundamental set of basic transcription machinery for ligand-dependent and -independent gene transcription (Roeder 2005). In addition, NR-mediated transcription requires NR coregulators that can interact directly or indirectly with NRs (Rosenfeld et al. 2006; Lonard and O'Malley 2007). NR coregulators were initially considered to act as bridges between the basic transcriptional machinery and NRs for efficient transcriptional controls. However, intensive studies over the past decade have revealed that many coregulators bear enzymatic and chromatin reorganization-related activities for histone modification, chromatin remodeling, and histone octamer transfer (Rosenfeld et al. 2006). Furthermore, biochemical approaches have shown that NR coregulators act in many cases as multisubunit complexes, which possess distinct enzymatic activities (Rachez et al. 1998; Kitagawa et al. 2003; Ohtake et al. 2007; Fujiki et al. 2009; Kim et al. 2009; Sawatsubashi et al. 2010; Baba et al. 2011). Sequential interactions of various NR coregulator complexes with NRs are assumed to foster dynamic changes in chromatin structure that facilitate transcriptional control (Kato et al. 2011; Fig. 1).

During the initial biochemical analyses of NR coregulator complexes, it was established that their coregulatory activity was strongly associated with histone acetylation/ deacetylation (Kamei et al. 1996; Heinzel et al. 1997; Nagy et al. 1997). Histone acetyltransferases (HATs) such as CREB binding protein (CBP)/p300 and GCN5 were consistently found to coactivate NRs, whereas histone deacetylases (HDACs) were shown to act as NR corepressors. However, recent progress has unveiled the role of histone methylation marks as "gatekeepers" of NR-mediated transcriptional controls (Garcia-Bassets et al. 2007). Thus, it is now suggested that histone methyltransferases (HMTs) and histone demethylases (HDMs) constitute a class of NR coregulators (Kato et al. 2011).

\section{HISTONE METHYLATION AND HMTS/HDMs}

The histone methylation mark is considered one of the most important "histone codes" that define the transcriptionally active or inactive chromatin state (Strahl and Allis 2000; Jenuwein and Allis 2001; Kouzarides 2007). Lysine residues in histone tails are mono-, di-, or tri-methylated by enzymes using $S$-adenosyl methionine (SAM) as the methyl group donor, whereas arginine 


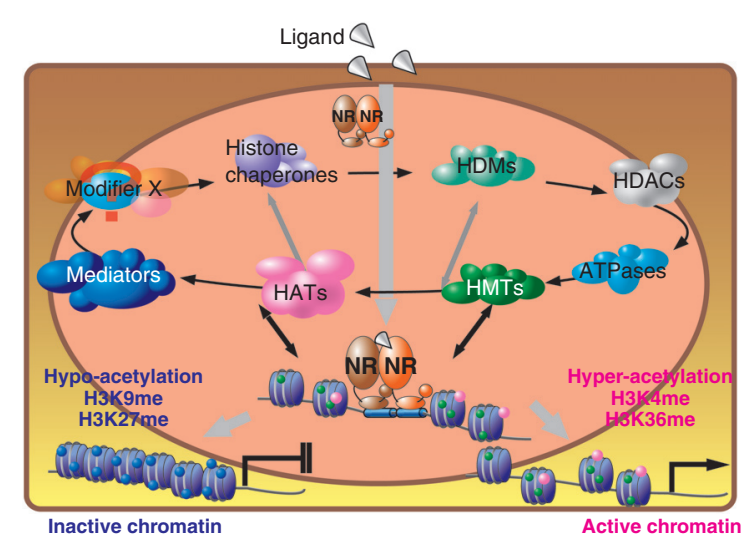

Figure 1. Coregulator complexes in nuclear receptor (NR)mediated gene regulation. NR-mediated transcriptional regulation is exerted through cyclical recruitment and dismissal of multiple coregulator complexes with distinct enzymatic activities, including histone acetyltransferases (HATs), histone methyltransferases (HMTs), histone deacetylases (HDACs), histone demethylases (HDMs), ATP-dependent chromatin remodelers, and histone chaperones. Other complexes with presently unidentified enzymatic activities are also assumed to be involved.

residues can be mono- or di- (asymmetric or symmetric) methylated (Martin and Zhang 2005). The identification of HMTs has demonstrated that histone methylations at specific sites are linked to specific chromatin states (Rea et al. 2000). For example, methylation at H3K4, $\mathrm{H} 3 \mathrm{~K} 36$, and H3K79 leads to chromatin activation in transcriptional controls, whereas methylation at $\mathrm{H} 3 \mathrm{~K} 9$, $\mathrm{H} 3 \mathrm{~K} 27$, and H4K20 inactivates the chromatin state (Martin and Zhang 2005). Moreover, the recent discovery of HDMs has converted the view of the histone methylation mark from a static mark into a dynamically reversible mark like histone acetylation (Cuthbert et al. 2004; Shi et al. 2004; Tsukada et al. 2006).

Histone methylation is catalyzed by three groups of HMTs: the SET (Su[var]3-9, Enhancer of zeste, Trithorax) domain-containing protein family, the protein arginine methyltransferase (PRMT) family, and the nonSET domain HMTs (Martin and Zhang 2005). Methyl moieties on histone proteins are removed by HDMs, which are categorized into three families: arginine deiminases, amine oxidases, and Jumonji C (JmjC) domaincontaining hydroxylases (Klose et al. 2006; Klose and Zhang 2007). More members have already been recorded for HMTs/HDMs than HATs/HDACs, with more substrate specificities. To date, several HMTs and HDMs have been reported to act as transcriptional coregulators for NRs (Kato et al. 2011). For example, coactivatorassociated arginine methyltransferase 1 (CARM1) coactivates NR transcription through H3R17 methylation (Chen et al. 1999), whereas lysine-specific demethylase 1 (LSD1) acts as a coactivator or corepressor for NRs, depending on the target sites for the demethylation reaction (Metzger et al. 2005; Garcia-Bassets et al. 2007; Yokoyama et al. 2008). Therefore, accumulating evidence suggests that regulation of histone methylation underlies ligand-dependent transcriptional controls by NRs. However, many aspects of NR transcriptional regulation remain to be uncovered. For example, the molecular link between the signaling pathways and NR coregulator function is only poorly understood. Furthermore, the mechanism by which the histone methylation state of the NR-targeted promoter is regulated by cellular signaling and the extracellular environment remains to be determined. Here, we present two examples of an emerging new paradigm in which the enzymatic activities of NR coregulator complexes are directly regulated through specific PTMs of their catalytic subunit, in response to cellular signaling and nutritional conditions. Finally, we will discuss how these findings are applicable to the diverse roles of NR coregulators in transmitting extracellular signals to the genome.

\section{ACTIVATION OF THE HISTONE METHYLTRANSFERASE MLL5 BY $O$-GICNACYLATION IN GRANULOCYTE DIFFERENTIATION}

Retinoic acid (RA) is essential for embryonic development, vision, and immune function (Duester 2008). RA is also known to be a potent inducer of cancer cell differentiation including acute promyeloid leukemia (APL) cells, and in fact RA is used for APL patients in clinical therapy (de The and Chen 2010). The effect of RA is exerted through a nuclear retinoic acid receptor (RAR) at the transcriptional level (Giguere 1994). However, the known RAR coregulators were unlikely to account for the diverse biological effects of RA.

In our efforts to search for new transcriptional regulators associated with RAR $\alpha$, the myeloid/lymphoid or mixed-lineage leukemia 5 (MLL5)-containing protein complex was identified in nuclear extracts of undifferentiated HL60 (APL-like) cells (Fig. 2A,B). Like the other MLL HMT members, MLL5 harbors a SET domain, but its sequence bears little homology with those of the other MLL members. When overexpressed in a transient luciferase assay in undifferentiated HL60 cells, MLL5 coactivated RAR $\alpha$, consistent with knockdown assays using short hairpin RNAs. When the SET domain (362-448 amino acid residues) was deleted $(\triangle \mathrm{SET})$ or inactivated (Y358A), RA-induced transactivation by means of RAR $\alpha$ was inhibited, suggesting that MLL5 acts as a novel coactivator for $\mathrm{RAR} \alpha$, presumably through its HMT activity (Fig. 2C). However, to our surprise, bacteria-derived recombinant MLL5 proteins possessed no histone methyltransferase activity. On the other hand, MLL5 prepared from HL60 cells exhibited strong activity with histone H3K4 (Figs. 2D and 3B).

To address this discrepancy in enzymatic activity, MLL5 complexes were isolated from an HL60 stable transformant expressing FLAG-tagged MLL5, and it appeared that there were two forms of MLL5-containing complexes with apparent masses of $\sim 1.5 \mathrm{MDa}$ (fractions 18-24, MLL5-L) and $\sim 600 \mathrm{kDa}$ (fractions 30-32, MLL5-S; Fig. 3A). As shown in Figure 3B, the MLL5-L fraction contained HMT activity, whereas activity was marginal in the MLL5-S fractions. 
A

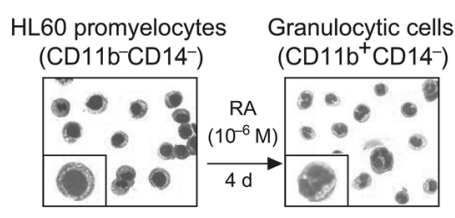

B

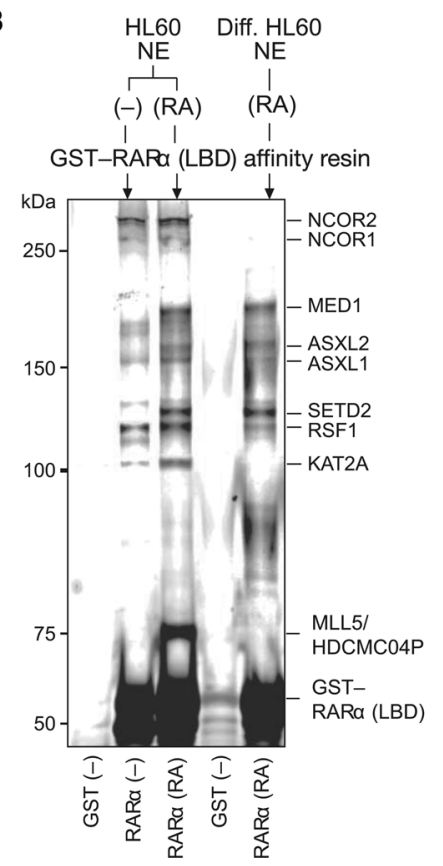

C

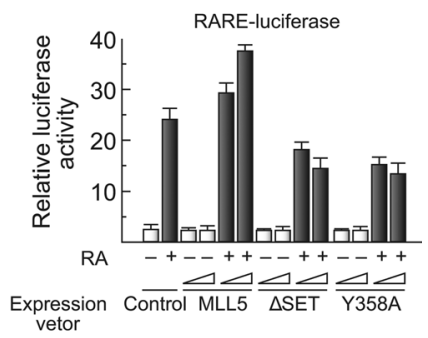

D

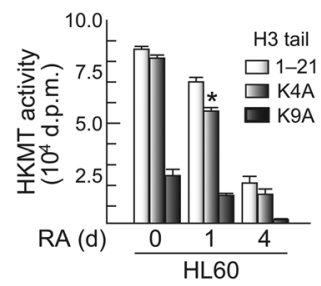

Figure 2. Identification of MLL5 as a coactivator of retinoic acid receptor $\alpha(\mathrm{RAR} \alpha)$. ( $A$ ) Giemsa-May-Grunwald staining and cellsurface marker of HL60 cells. (B) Outline (top) and silver-staining analysis (bottom) of the purification using glutathione $S$-transferase (GST)-fused RAR $\alpha$ (LBD) as bait. Diff. HL60, differentiation. (C) Luciferase assay of mixed-lineage leukemia 5 (MLL5) function in RAR-mediated transcription. RARE, RA response element. (D) RAR $\alpha$-associating HMT activities during RA-induced differentiation. The anti-RAR $\alpha$ immunoprecipitates from differentiating cells were used for in vitro HMT assays with H3 tail peptides (1-21) and the indicated point-mutated peptides. ${ }^{*} P<0.05$ versus the activity for the $1-21$ peptides. Error bars, means and S.D. $(n=3)$. d.p.m., disintegrations per minute. (Reprinted, with permission, from Fujiki et al. 2009.)

Mass spectrometric analysis revealed that the MLL5-L and MLL5-S complexes shared five components. Importantly, the large complex (possessing HMT activity) contained two additional subunits, host cell factor-1 amino-terminal subunit (HCFC1) and $O$-linked $N$-acetylglucosamine (GlcNAc) transferase (OGT) (Fig. 3C). The presence of OGT suggested that it activated HMT function through GlcNAcylation of a complex subunit. In fact, recombinant OGT protein was able to GlcNAcylate recombinant MLL5 protein at Thr440. Moreover, GlcNAcylated MLL5 exhibited robust H3K4 HMT activity in vitro (Fig. 3D). When the GlcNAcylation moieties of the complex were de-GlcNAcylated by hexosaminidase $(O$-GlcNAcase) treatment, the purified large MLL5 complex completely lost HMT activity and was converted to the MLL5-S form (Fig. 3E). Thus, GlcNAcylation of MLL5 appeared necessary to assemble a large and enzymatically active form of the MLL5 complex.

MLL5 GlcNAcylation and its HMT activity were dependent on sufficient levels of nuclear uridine diphosphate (UDP)-GlcNAc, a substrate of OGT.
UDP-GlcNAc is derived from extracellular glucose through the cellular hexosamine biosynthesis pathway (HBP). When cells were cultured in high glucose (10$30 \mathrm{mM}$ ) medium or treated with a GlcNAcase inhibitor (PUGNAc, $\sim 150 \mu \mathrm{M}$ ) to fully induce nuclear protein GlcNAcylation, both treatments effectively potentiated RA-induced differentiation of HL60 into granulocytes, supporting our findings that MLL5 is a GlcNAcylationdependent RAR coactivator (Fig. 4).

Of interest, the endogenous HMT activity of MLL5 was much lower in an HL60 subline (HL60-R2 cells) that was resistant to RA-induced cellular differentiation through an unknown mechanism. However, treatment with an $O$-GlcNAcase inhibitor, PUGNAc, restored responses to RA and RA-induced methylation of histone H3K4. The level of MLL5 GlcNAcylation in HL60-R2 cells was lower than in HL60 cells, and untreated HL60-R2 cells contained very low levels of intracellular glucose and high $O$-GlcNAcase activity in comparison to HL60 cells, indicating nuclear hypo-GlcNAcylation in HL60-R2 cells. 
A

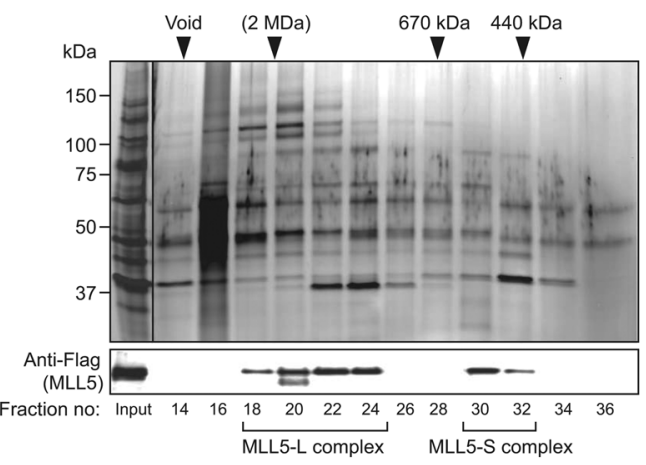

B

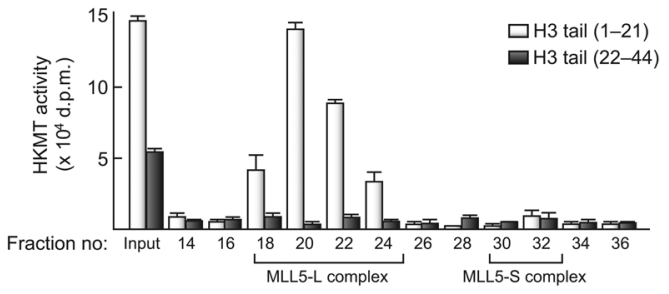

C

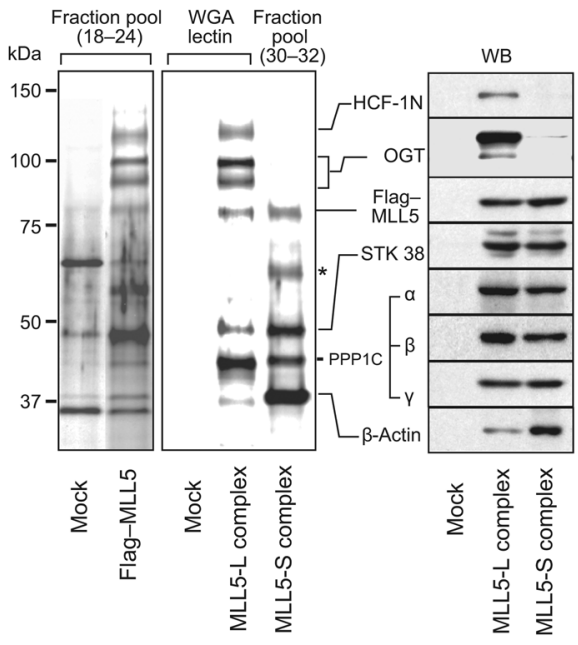

$\mathbf{E}$

D
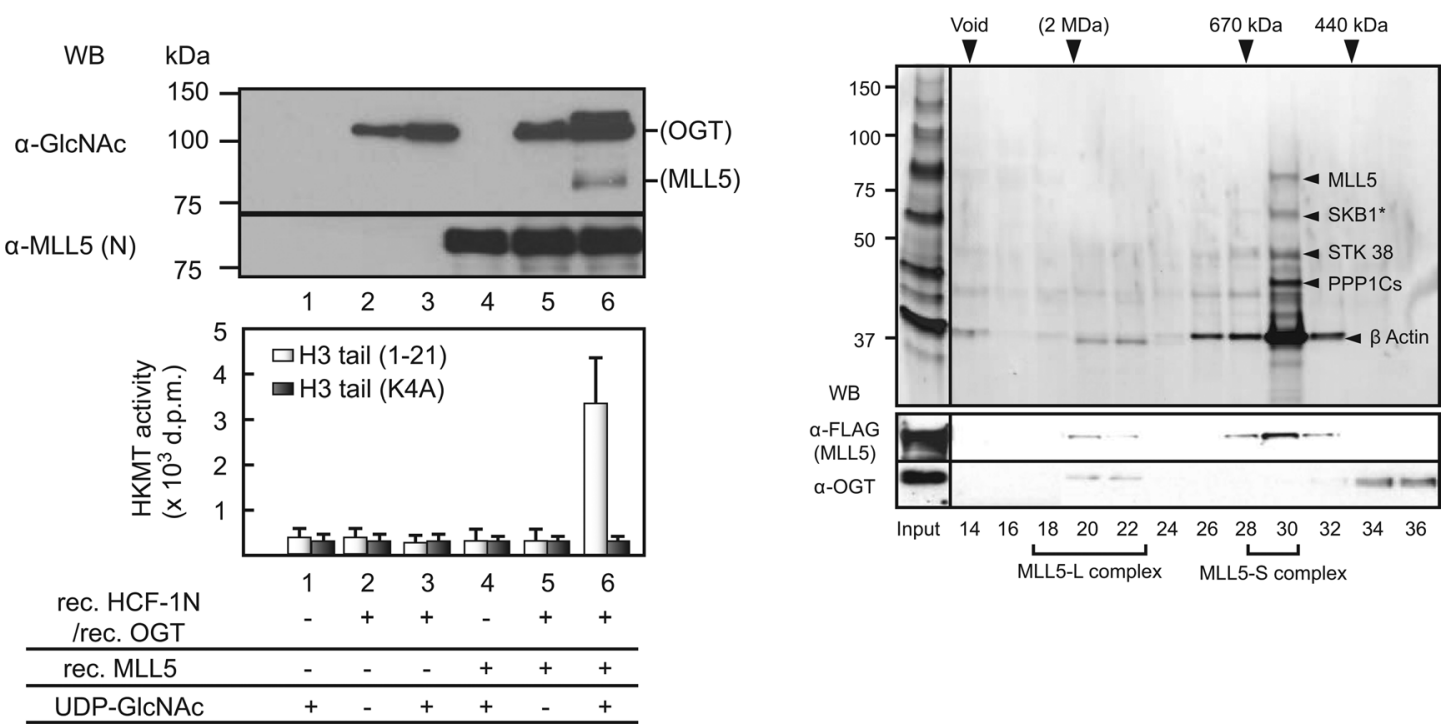

Figure 3. MLL5 complex as a GlcNAcylation-dependent HMT complex. (A) Silver-staining and western blot analysis of the gelfiltrated FLAG-MLL5 immunocomplex. (B) HMT activities of the gel-filtrated fractions using the indicated tail peptides. (C) Silverstaining and western blot analysis of the MLL5-L and the MLL5-S complexes. (D) In vitro GlcNAcylation levels of the recombinant proteins were analyzed by western blot with $\alpha$-GlcNAc (top). The GlcNAcylated samples were subjected to an in vitro HMT assay using H3 tail (1-21) peptides (bottom). (E) The $\alpha$-FLAG affinity-purified MLL5 complex was treated with recombinant $O$-GlcNAcase and subjected to fractionation by gel filtration chromatography. Silver-staining and western blot analysis with the indicated antibodies for each fraction are shown. (Reprinted, with permission, from Fujiki et al. 2009.)

Thus, these studies demonstrate that the function of an HMT complex is regulated by $O$-GlcNAcylation, and that monoglycosylation is a vital PTM in gene regulation (Fujiki et al. 2009).

\section{HISTONE DEMETHYLASE IS ACTIVATED IN RESPONSE TO FASTING SIGNALS}

During a biochemical survey of transcriptional coregulators supporting unliganded farnesoid X receptor (FXR) from HepG2 hepatoma cells, we purified a coactivator complex (Fig. 5A). The association with FXR was significantly increased when the cells were pretreated with forskolin (FSK), a potent protein kinase A (PKA) activator. Mass spectrometry analysis of the purified complex identified three subunits: plant homeodomain (PHD) finger two (PHF2), AT-rich interactive domain 5B (ARID5B) isoform $\alpha$, and ARID5B isoform $\beta$ (Fig. 5B). PHF2 contains PHD and JmjC domains. Unlike several other JmjC domain-containing histone demethylases (e.g., Jumonji/ARID domain-containing protein 1C [JARID1C]), PHF2 lacks the ARID domain (Fig. 5C). 

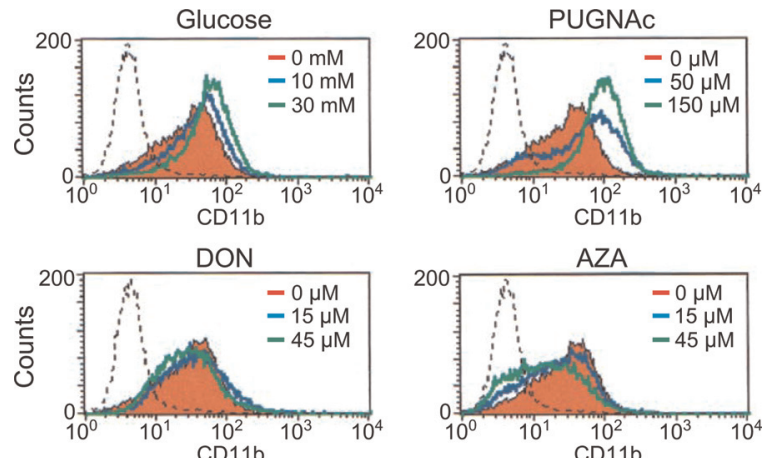

Figure 4. GlcNAcylation of MLL5 facilitates RA-induced granulopoiesis. Effect of cellular GlcNAcylation on RA-induced granulopoiesis. HL60 cells, exposed to the indicated reagents, were analyzed by flow cytometry. The dashed lines show RA-untreated control. (Reprinted, with permission, from Fujiki et al. 2009.)
As JmjC domain-containing proteins catalyze lysine demethylation, we investigated the histone lysine demethylation activity of the purified PHF2 complex. Of interest, like MLL5, histone demethylase activity was not detected in recombinant PHF2 protein, but the PHF2 complex purified from nuclear extracts demethylated dimethylated Lys 9 on histone $\mathrm{H} 3$ (H3K9me2) (Fig. 5D).

We then determined whether the demethylase activity of PHF2 was PKA-dependent by using PHF2 protein purified from $293 \mathrm{~F}$ cells as well as recombinant protein. Purified PHF2 protein exhibited H3K9me2 demethylase activity when phosphorylated by PKA in vitro (Fig. 5E). Conversely, in vitro dephosphorylation of phosphorylated PHF2 by bacterial alkaline phosphatase (BAP) negated the demethylase activity, and ARID5B was dispensable for the enzymatic activity of PHF2 in vitro. Consistent with the in vitro analyses, H3K9me2
A

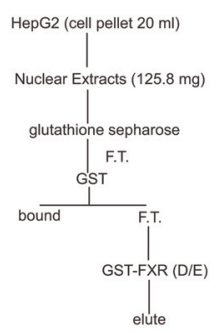

D

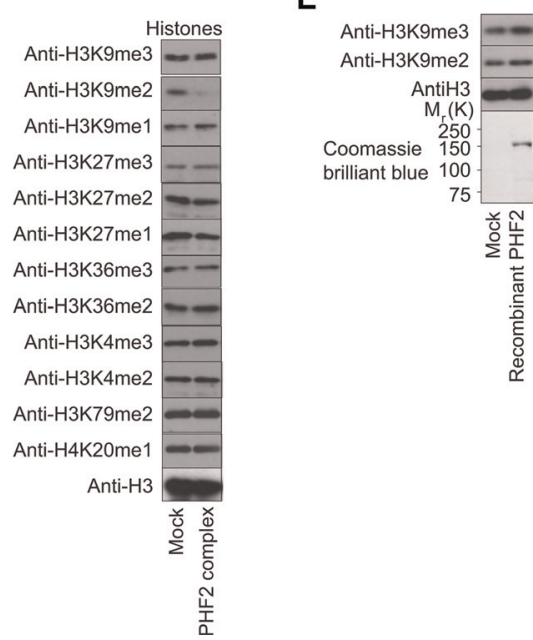

B

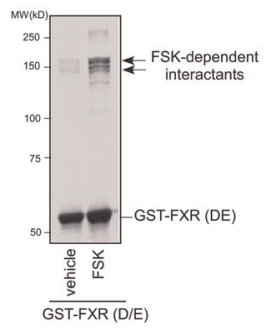

C

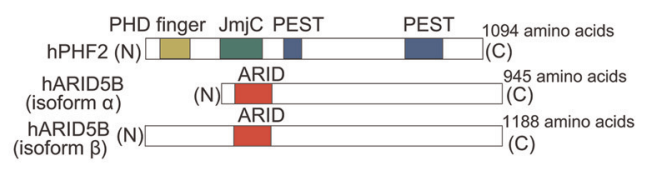

G

Figure 5. Identification of the PHF2-ARID5B histone H3K9me2 demethylase complex. $(A)$ Purification of signal-dependent GST-FXR-associated proteins from HepG2 cells. Nuclear extracts from FSK-treated HepG2 cells were loaded on GST-FXR-bound glutathione Sepharose beads. The bound proteins were analyzed by silver staining. $(B)$ The eluted complexes were separated by glycerol density gradients and isolated complex were silver stained, and each protein was identified by MALDI-TOF/MS and fingerprinting. (C) A schematic representation of domains of PHF2 and ARID5B. (D) The PHF2 complex was incubated with purified native histones for demethylation reaction, and each histone modification was determined by western blotting as indicated. $(E)$ Recombinant PHF2 protein is catalytically inactive. Recombinant PHF2 protein (lower panel) was purified and subjected to in vitro demethylation assay (upper panel). $(F)$ In vitro histone demethylation assay. Purified PHF2 protein or PHF2 mutants were incubated with either PKA or BAP as indicated, and in vitro demethylation assays were carried out with/without ARID5B protein. $(G) \mathrm{H} 3 \mathrm{~K} 9 \mathrm{me} 2$ demethylation activity of PHF2-ARID5B is FSK-dependent in vivo. 293F cells transfected with wild-type or 4A mutant were treated with FSK for $6 \mathrm{~h}$. Scale bars, $40 \mu \mathrm{m}$. (Reprinted, with permission, from Baba et al. 2011.) 
demethylation in cells overexpressing PHF2-ARID5B was significantly enhanced by the FSK treatment, but 4SA, a PHF2 phosphorylation negative mutant, was not activated by FSK (Fig. 5F). Together, these findings suggest that PKA-mediated phosphorylation induces the demethylase activity of PHF2, unlike the other characterized jmjC demethylases exhibiting constitutive enzymatic activities both in vivo and in vitro.

Although ARID5B was dispensable for PHF2 enzymatic activity in vitro, ARID5B was required for promoter targeting of PHF2 and H3K9me2 demethylation in vivo. Interestingly, a specific PHF2 mutant (H249A) lacked a conserved histidine essential for its lysine demethylation activity and was unable to anchor PHF2-ARID5B on the target gene promoter. Therefore, we reasoned that a lysine demethylation event occurred before chromatin association. In fact, ARID5B was lysine methylated in vivo. Moreover, using an anti-ARID5B-K336me2 antibody, we found that PHF2 promoted demethylation of ARID5B at Lys 336 (Fig. 6A). Furthermore, the ARID5B-PHF2 complex was recruited to Pepck promoter sequences only when cells were treated with FSK (Fig. 6B). Several other JmjC domain-containing demethylases such as JARID1c possess both the JmjC and the ARID domains within a single molecule. Thus, segregation of these two domains into separate subunits might achieve greater sensitivity of the PHF2-ARID5B complex to extracellular signaling. Thus, in vivo, demethylated ARID5B appears to be required for the recruitment of the PHF2-ARID5B complex to targeted promoters.

Finally, the physiological relevance of the proposed promoter targeting by PHF2-ARID5B was examined in animals under fasting conditions in which the PKA signal was activated by glucagon. In fasting mice, co-recruitment of PHF2 with ARID5B was detected in the promoter regions of Pepck and G6Pase in liver with the expected decreases in $\mathrm{H} 3 \mathrm{~K} 9 \mathrm{me} 2$ modification (Fig. 6C).

Taken together, we presume that PKA-dependent activation of this histone demethylase complex permits PKA signaling to activate target genes, and mediate the fasting response in the liver to activate the gluconeogenic program (Baba et al. 2011).

\section{CONCLUSIONS}

Target gene regulation by NRs is based on discrete systems at each transcriptional step, such as transcriptional initiation, elongation, termination, coregulator
A

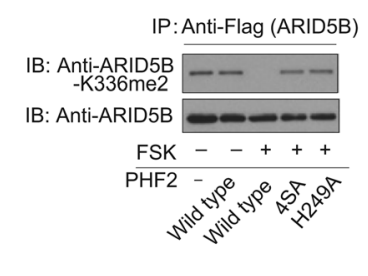

B

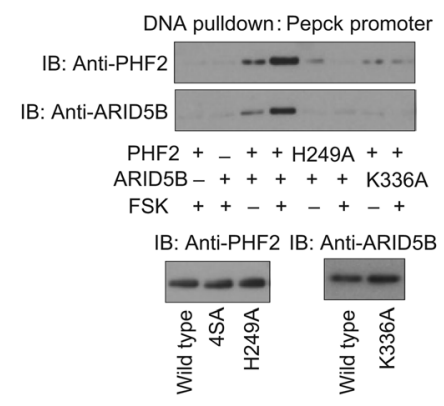

C
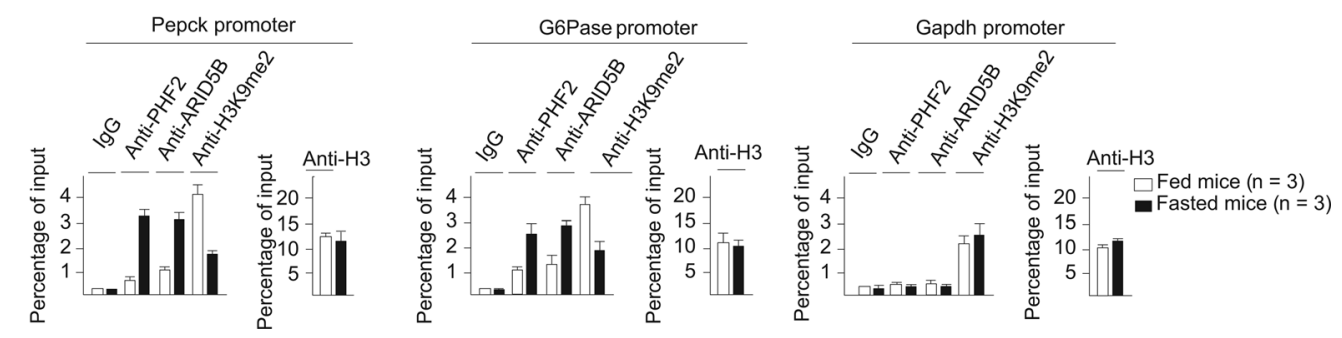

Figure 6. ARID5B directs PKA-dependent promoter targeting of PHF2. (A) Demethylation of ARID5B is dependent on the catalytic activity of PHF2 in hepatocytes as revealed by the specified anti-ARID5B-K336me2 antibody. (B) DNA pulldown assay. The demethylase activity of PHF2 and the ARID domain of ARID5B are required for promoter DNA binding of PHF2-ARID5B in 293F cells. Cell lysates were mixed with avidin beads, which were bound to biotin-conjugated oligonucleotides bearing the indicated promoter sequence. The bound protein was detected by western blotting. There were similar levels of expression of PHF2-ARID5B and their derivatives (lower panels). (C) Livers from either fasting or fed mice $(n=3)$ were subjected to ChIP analysis using anti-PHF2, anti-ARID5B, anti-H3K9me2, or control IgG and anti-H3 antibodies. Fasting induces recruitment of PHF2-ARID5B and reduction of the H3K9me2 mark in Pepck and G6Pase promoters, but not the Gapdh promoter. Data shown are averages \pm S.D. of the independent experiments. (Reprinted, with permission, from Baba et al. 2011.) 


\section{REGULATED HMT/HDM COMPLEXES FOR NR TRANSCRIPTION}

recruitment, histone modification, chromatin remodeling, and coregulator dissociation. Here, we highlighted our findings that histone methylation-modifying activities of novel NR coregulator complexes are regulated via two PTMs, GlcNAcylation and phosphorylation (Fujiki et al. 2009; Baba et al. 2011). Overall, these findings suggest that cells use transcriptional activation of target genes to regulate the transmission of signals from extracellular stimuli to the genome (Fig. 7).

\section{NR Coregulators as Nutrient Sensors}

GlcNAcylation of MLL5 was dependent, at least in part, on extracellular glucose levels and the overall cellular energetic state. Phosphorylation of PHF2 was induced by activated PKA signaling under fasting conditions in animals. Thus, these factors appear to serve as nutrientsensing epigenetic determinants through their regulation of histone methylation marks in a PTM-dependent manner. These mechanisms suggest a new paradigm of gene regulation in which histone modification enzymes/complexes are responsive to the nutritional state as well as intracellular levels of metabolites such as acetyl-CoA, NAD ${ }^{+}$, and SAM (Borrelli et al. 2008; Gibson and Kraus 2011).

The uptake of these metabolites into the nucleus and consequent use by histone-modifying enzymes as substrates are under intensive study. Recently, methionine adenosyltransferase II alpha (MATII $\alpha$ ) was reported to provide SAM for methylation reactions by HMTs (Katoh et al. 2011), and acetyl-CoA synthetase (ACS) was shown to be an acetyl-CoA supplier for HATs (Takahashi et al. 2006). Although the UDP-GlcNAc supplier in the nucleus has not yet been identified, from the present findings, its presence is highly likely. Thus, not all, but certain NR coregulator complexes appear to integrate extracellular and intracellular nutrient conditions through various PTMs to fine tune chromatin reorganization at target gene promoters.

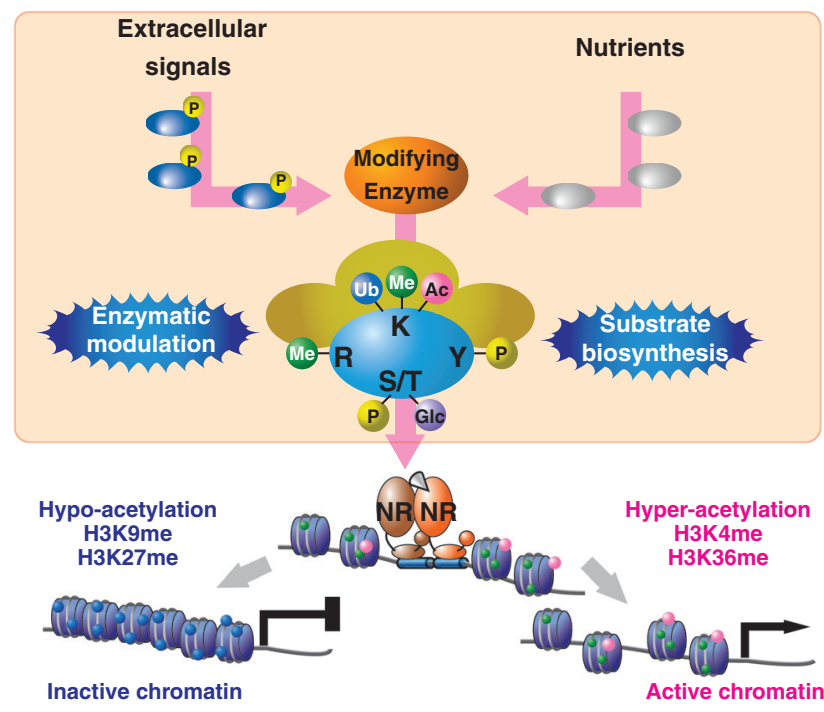

\section{Regulation of NR Transcriptional Activities via PTMs}

The transcriptional activities of liganded NRs are mainly governed by ligand binding followed by conformational change of the carboxy-terminal $\alpha$-helix (Helix12) in the LBD together with sequential coregulator switching (Nichols et al. 1998). In addition, the transcriptional activities of NRs are modulated by other cellular signaling pathways. Cross talk between an NR and membrane receptor-mediated signals was first observed in the phosphorylation-induced activation of estrogen receptor $\alpha(\mathrm{ER} \alpha)$ by a MAPK-mediated signal (Kato et al. 1995). Such signal-induced regulation by protein phosphorylation was also observed for HAT-type NR coregulators such as steroid receptor coactivator-3 (SRC-3) (Wu et al. 2002; Wu et al. 2004), and NCoR (Hermanson et al. 2002). They undergo multiple phosphorylations that affect their functionality through modulation of protein stability and protein-protein interactions. From the two examples illustrated in the present review, this system seems applicable to HMT/HDM-type NR coregulator complexes. Of note, these are the first observations that specific PTMs control the enzymatic activities of NR coregulator complexes. Thus, for other putative histone modifying enzymes that are inactive as single subunits, PTMs of the subunits could trigger both complex assembly and enzymatic activation.

PTMs are efficient biological signals for expanding the genetic code and they play key roles in regulating various processes at the cellular level, including transcription (Walsh et al. 2005). Although the significance of protein PTMs in gene regulation is increasingly being shown, only limited numbers of PTMs have been characterized. For example, in histone proteins, eight distinct PTMs (acetylation, methylation [K and $\mathrm{R}]$, phosphorylation, ubiquitilation, SUMOylation, ADP ribosylation, deimination, and proline isomerization) are known (Kouzarides 2007). The impact of GlcNAcylation in MLL5-mediated
Figure 7. PTMs integrate extracellular signaling and nutrient signaling via coregulator complexes. Extracellular and nutrient signals modulate the activities of modifying enzymes. These enzymes covalently modify the subunits (and enzymatic activities) of the coregulator complexes, thereby evoking the complexes' potential functions. 
histone methylation implies that it, too, is a vital PTM for other nuclear events such as histone modification and chromatin remodeling. This idea could be further expanded to other PTMs, because more than 500 PTMs have been described to date (Ahrne et al. 2010). Therefore, it is of great interest to identify other PTMs that are important for NR coregulator functions.

\section{Concluding Remarks}

In light of our findings, we conclude that certain NR coregulator complexes are "sensors" that integrate cellular signals into precise and proper gene regulation through PTMs. Specifically, protein GlcNAcylation requires further characterization as a vital PTM to modulate protein function in cellular and nuclear events. Thus far, specific HMTs and HDMs have been successfully documented to act as PTM-dependent NR coregulators, but additional classes of PTM-dependent NR coregulators, such as chromatin remodelers, histone chaperons and other previously unidentified enzymes, are expected to be demonstrated in the near future.

\section{ACKNOWLEDGMENT}

We thank M. Yamaki for manuscript preparation.

\section{REFERENCES}

Ahrne E, Muller M, Lisacek F. 2010. Unrestricted identification of modified proteins using MS/MS. Proteomics 10: $671-686$.

Baba A, Ohtake F, Okuno Y, Yokota K, Okada M, Imai Y, Ni M, Meyer CA, Igarashi K, Kanno J, et al. 2011. PKA-dependent regulation of the histone lysine demethylase complex PHF2-ARID5B. Nat Cell Biol 13: 669-676.

Bamberger CM, Schulte HM, Chrousos GP. 1996. Molecular determinants of glucocorticoid receptor function and tissue sensitivity to glucocorticoids. Endocr Rev 17: 245-261.

Borrelli E, Nestler EJ, Allis CD, Sassone-Corsi P. 2008. Decoding the epigenetic language of neuronal plasticity. Neuron 60: 961-974.

Chen D, Ma H, Hong H, Koh SS, Huang SM, Schurter BT, Aswad DW, Stallcup MR. 1999. Regulation of transcription by a protein methyltransferase. Science 284: 2174-2177.

Couse JF, Korach KS. 1999. Estrogen receptor null mice: What have we learned and where will they lead us? Endocr Rev 20: $358-417$.

Cuthbert GL, Daujat S, Snowden AW, Erdjument-Bromage H, Hagiwara T, Yamada M, Schneider R, Gregory PD, Tempst $\mathrm{P}$, Bannister AJ, et al. 2004. Histone deimination antagonizes arginine methylation. Cell 118: 545-553.

de The H, Chen Z. 2010. Acute promyelocytic leukaemia: Novel insights into the mechanisms of cure. Nat Rev Cancer 10: 775-783.

Duester G. 2008. Retinoic acid synthesis and signaling during early organogenesis. Cell 134: 921-931.

Fujiki R, Chikanishi T, Hashiba W, Ito H, Takada I, Roeder RG, Kitagawa H, Kato S. 2009. GlcNAcylation of a histone methyltransferase in retinoic-acid-induced granulopoiesis. Nature 459: 455-459.

Garcia-Bassets I, Kwon YS, Telese F, Prefontaine GG, Hutt KR, Cheng CS, Ju BG, Ohgi KA, Wang J, Escoubet-Lozach L, et al. 2007. Histone methylation-dependent mechanisms impose ligand dependency for gene activation by nuclear receptors. Cell 128: 505-518.
Gibson BA, Kraus WL. 2011. Small molecules, big effects: A role for chromatin-localized metabolite biosynthesis in gene regulation. Mol Cell 41: 497-499.

Giguere V. 1994. Retinoic acid receptors and cellular retinoid binding proteins: Complex interplay in retinoid signaling. Endocr Rev 15: 61-79.

Giguere V. 1999. Orphan nuclear receptors: From gene to function. Endocr Rev 20: 689-725.

Heinzel T, Lavinsky RM, Mullen TM, Soderstrom M, Laherty CD, Torchia J, Yang WM, Brard G, Ngo SD, Davie JR, et al. 1997. A complex containing N-CoR, $\mathrm{mSin} 3$ and histone deacetylase mediates transcriptional repression. Nature 387: $43-48$.

Hermanson O, Jepsen K, Rosenfeld MG. 2002. N-CoR controls differentiation of neural stem cells into astrocytes. Nature 419: $934-939$.

Jenuwein T, Allis CD. 2001. Translating the histone code. Science 293: 1074-1080.

Kamei Y, Xu L, Heinzel T, Torchia J, Kurokawa R, Gloss B, Lin SC, Heyman RA, Rose DW, Glass CK, et al. 1996. A CBP integrator complex mediates transcriptional activation and AP-1 inhibition by nuclear receptors. Cell 85: 403414.

Kato S, Endoh H, Masuhiro Y, Kitamoto T, Uchiyama S, Sasaki H, Masushige S, Gotoh Y, Nishida E, Kawashima H, et al. 1995. Activation of the estrogen receptor through phosphorylation by mitogen-activated protein kinase. Science 270: $1491-1494$

Kato S, Yokoyama A, Fujiki R. 2011. Nuclear receptor coregulators merge transcriptional coregulation with epigenetic regulation. Trends Biochem Sci 36: 272-281.

Katoh Y, Ikura T, Hoshikawa Y, Tashiro S, Ito T, Ohta M, Kera Y, Noda T, Igarashi K. 2011. Methionine adenosyltransferase II serves as a transcriptional corepressor of Maf oncoprotein. Mol Cell 41: 554-566.

Kim MS, Kondo T, Takada I, Youn MY, Yamamoto Y, Takahashi S, Matsumoto T, Fujiyama S, Shirode Y, Yamaoka I, et al. 2009. DNA demethylation in hormone-induced transcriptional derepression. Nature 461: 1007-1012.

King-Jones K, Thummel CS. 2005. Nuclear receptors-A perspective from Drosophila. Nat Rev Genet 6: 311-323.

Kitagawa H, Fujiki R, Yoshimura K, Mezaki Y, Uematsu Y, Matsui D, Ogawa S, Unno K, Okubo M, Tokita A, et al. 2003. The chromatin-remodeling complex WINAC targets a nuclear receptor to promoters and is impaired in Williams syndrome. Cell 113: 905-917.

Klose RJ, Zhang Y. 2007. Regulation of histone methylation by demethylimination and demethylation. Nat Rev Mol Cell Biol 8: $307-318$.

Klose RJ, Kallin EM, Zhang Y. 2006. JmjC-domain-containing proteins and histone demethylation. Nat Rev Genet 7: $715-727$.

Kouzarides T. 2007. Chromatin modifications and their function. Cell 128: 693-705.

Lonard DM, O’Malley BW. 2007. Nuclear receptor coregulators: Judges, juries, and executioners of cellular regulation. Mol Cell 27: 691-700.

Mangelsdorf DJ, Thummel C, Beato M, Herrlich P, Schutz G, Umesono K, Blumberg B, Kastner P, Mark M, Chambon P, et al. 1995. The nuclear receptor superfamily: The second decade. Cell 83: 835-839.

Martin C, Zhang Y. 2005. The diverse functions of histone lysine methylation. Nat Rev Mol Cell Biol 6: 838-849.

Metzger E, Wissmann M, Yin N, Muller JM, Schneider R, Peters AH, Gunther T, Buettner R, Schule R. 2005. LSD1 demethylates repressive histone marks to promote androgenreceptor-dependent transcription. Nature 437: 436-439.

Nagy L, Kao HY, Chakravarti D, Lin RJ, Hassig CA, Ayer DE, Schreiber SL, Evans RM. 1997. Nuclear receptor repression mediated by a complex containing SMRT, mSin3A, and histone deacetylase. Cell 89: 373-380.

Nichols M, Rientjes JM, Stewart AF. 1998. Different positioning of the ligand-binding domain helix 12 and the F domain of the 
estrogen receptor accounts for functional differences between agonists and antagonists. EMBO J 17: 765-773.

Ohtake F, Baba A, Takada I, Okada M, Iwasaki K, Miki H, Takahashi S, Kouzmenko A, Nohara K, Chiba T, et al. 2007. Dioxin receptor is a ligand-dependent E3 ubiquitin ligase. Nature 446: $562-566$.

Rachez C, Suldan Z, Ward J, Chang CP, Burakov D, ErdjumentBromage H, Tempst P, Freedman LP. 1998. A novel protein complex that interacts with the vitamin D3 receptor in a ligand-dependent manner and enhances VDR transactivation in a cell-free system. Genes Dev 12: 1787-1800.

Rea S, Eisenhaber F, O'Carroll D, Strahl BD, Sun ZW, Schmid M, Opravil S, Mechtler K, Ponting CP, Allis CD, et al. 2000. Regulation of chromatin structure by site-specific histone $\mathrm{H} 3$ methyltransferases. Nature 406: 593-599.

Roeder RG. 2005. Transcriptional regulation and the role of diverse coactivators in animal cells. FEBS Lett 579: 909-915.

Rosenfeld MG, Lunyak VV, Glass CK. 2006. Sensors and signals: A coactivator/corepressor/epigenetic code for integrating signal-dependent programs of transcriptional response. Genes Dev 20: 1405-1428.

Sawatsubashi S, Murata T, Lim J, Fujiki R, Ito S, Suzuki E, Tanabe M, Zhao Y, Kimura S, Fujiyama S, et al. 2010. A histone chaperone, DEK, transcriptionally coactivates a nuclear receptor. Genes Dev 24: 159-170.

Shi Y, Lan F, Matson C, Mulligan P, Whetstine JR, Cole PA, Casero RA. 2004. Histone demethylation mediated by the nuclear amine oxidase homolog LSD1. Cell 119: 941-953.

Strahl BD, Allis CD. 2000. The language of covalent histone modifications. Nature 403: 41-45.

Takahashi H, McCaffery JM, Irizarry RA, Boeke JD. 2006. Nucleocytosolic acetyl-coenzyme a synthetase is required for histone acetylation and global transcription. Mol Cell 23: $207-217$.

Tsukada Y, Fang J, Erdjument-Bromage H, Warren ME, Borchers CH, Tempst P, Zhang Y. 2006. Histone demethylation by a family of JmjC domain-containing proteins. Nature 439: $811-816$

Walsh CT, Garneau-Tsodikova S, Gatto GJ Jr. 2005. Protein posttranslational modifications: The chemistry of proteome diversifications. Angew Chem Int Ed Engl 44: $7342-7372$.

Wu RC, Qin J, Hashimoto Y, Wong J, Xu J, Tsai SY, Tsai MJ, O'Malley BW. 2002. Regulation of SRC-3 (pCIP/ACTR/ AIB-1/RAC-3/TRAM-1) coactivator activity by I $\kappa$ B kinase. Mol Cell Biol 22: 3549-3561.

Wu RC, Qin J, Yi P, Wong J, Tsai SY, Tsai MJ, O’Malley BW. 2004. Selective phosphorylations of the SRC-3/AIB1 coactivator integrate genomic responses to multiple cellular signaling pathways. Mol Cell 15: 937-949.

Yokoyama A, Takezawa S, Schule R, Kitagawa H, Kato S. 2008. Transrepressive function of TLX requires the histone demethylase LSD1. Mol Cell Biol 28: 3995-4003. 


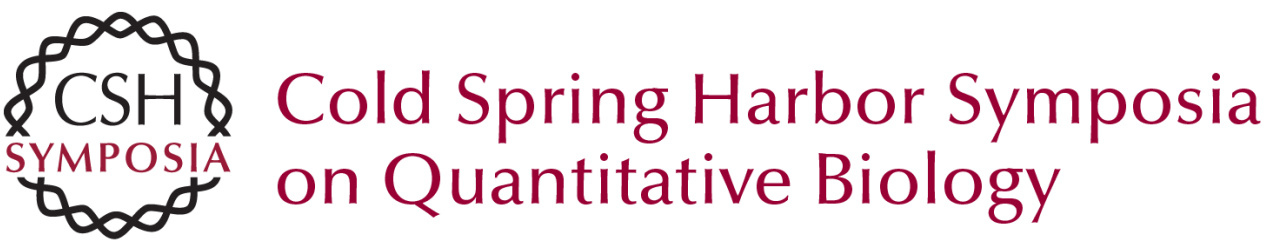

\section{Regulated Histone Methyltransferase and Demethylase Complexes in the Control of Genes by Nuclear Receptors}

A. Yokoyama, R. Fujiki, F. Ohtake, et al.

Cold Spring Harb Symp Quant Biol 2011 76: 165-173 originally published online September 2, 2011

Access the most recent version at doi:10.1101/sqb.2011.76.010736

References This article cites 47 articles, 9 of which can be accessed free at: http://symposium.cshlp.org/content/76/165.full.html\#ref-list-1

\section{License}

Email Alerting Receive free email alerts when new articles cite this article - sign up in Service the box at the top right corner of the article or click here. 